\title{
Nonparametric estimation for the ruin probability in a Lévy risk model under low-frequency observation
}

\author{
Zhimin Zhang*a, Hailiang Yang ${ }^{\mathrm{b}}$ \\ ${ }^{a}$ College of Mathematics and Statistics, Chongqing University, Chongqing, 401331, PR China \\ ${ }^{b}$ Department of Statistics and Actuarial Science, \\ The University of Hong Kong, Pokfulam road, Hong Kong
}

\begin{abstract}
In this paper, we propose a nonparametric estimator for the ruin probability in a spectrally negative Lévy risk model based on low-frequency observation. The estimator is constructed via the Fourier transform of the ruin probability. The convergence rates of the estimator are studied for large sample size. Some simulation results are also given to show the performance of the proposed method when the sample size is finite.
\end{abstract}

Keywords: Lévy risk model, Ruin probability, Estimator, Fourier transform.

\section{Introduction}

In actuarial science, the surplus process of an insurance company is frequently described by the classical compound Poisson risk model (also called Cramér-Lundberg model), and one of the main topics in ruin theory is the ruin probability. In the past two decades, a number of methods have be proposed to study the ruin probability, among which are integro-differential equation technique, renewal theory, Laplace transform, random walk arguments, martingale theory, heavy-tailed asymptotic estimations and so on. Various applications of the above mentioned methods can be found in the nice monograph by Asmussen and Albrecher (2010).

The analytic (or probabilistic) approach is heavily dependent on the knowledge of the risk model, such as the premium rate, the inter-claim time distribution and the claim size distribution. However, in practice, instead of having precise information on the risk model, it is more likely that only some observed data on the surplus process is available. From this point of view, statistical methodology is of great importance. For the estimation of ruin probability, many (semi-)parametric and nonparametric estimators have been proposed in the literature. See, for example, Frees (1986), Hipp (1989), Croux

${ }^{*}$ Corresponding author. E-mail address: zmzhang@cqu.edu.cn (Z. Zhang) 
and Vervaerbeke (1990), Pitts (1994) and Politis (2003), Shimizu (2009). For more recent development on this topic, we refer the interested readers to Masiello (2012), Zhang et al. (2012) and Zhang and Yang (2013) on estimating the ruin probability, and Shimizu (2012) on estimating the general Gerber-Shiu function.

In financial market, high frequency trading exists and a lot of high frequency trading data can be used to make statistical inference of the law of financial market. However, for an insurance company, it is often the case that only daily or weekly book data on the surplus level exists. Hence, it is very useful and interesting to study how to estimate the risk measure for an insurance surplus flow based on low frequency data. In the present work, we study how to estimate the ruin probability in a classes of spectrally negative Lévy risk models described below. To the best of our knowledge, this problem has not been solved in the literature.

Let $u \geq 0$ be the initial surplus of an insurance company. The surplus level at time $t$ is given by $u+X_{t}$, where $X=\left\{X_{t}, t \geq 0\right\}$ is a spectrally negative Lévy process. Suppose that $X_{0}=0$ and $X_{t}$ has characteristic function

$$
\phi_{X_{t}}(s):=\mathbb{E} e^{i s X_{t}}=e^{t \Psi(s)},
$$

with characteristic exponent

$$
\Psi(s)=i c s-\frac{\sigma^{2}}{2} s^{2}+\int_{0}^{\infty}\left(e^{-i s x}-1\right) \nu(d x),
$$

where $c>0, \sigma^{2} \geq 0$, and $\nu$ is a Lévy measure supported on $(0, \infty)$ satisfying the usual condition

$$
\int_{0}^{\infty}\left(1 \wedge x^{2}\right) \nu(d x)<\infty
$$

and the net profit condition

$$
c>\mu_{1}:=\int_{0}^{\infty} x \nu(d x) .
$$

Huzak et al. (2004) considered a more general perturbed risk model defined by

$$
R_{t}=u+c t-C_{t}+P_{t}, \quad t \geq 0,
$$

where $C_{t}$ is a pure-jump Lévy process with only positive jumps, representing the total claims up to time $t$, and $P_{t}$ is a spectrally negative Lévy process with zero mean, representing the perturbation. Note that the Lévy risk model $X_{t}$ in this paper is a special case of (1.2), since in fact we have replaced $P_{t}$ by a Brownian motion. However, such special setting is not restrictive, because we can always remove the linear drift and the jump components in $P_{t}$ to the premium income and the aggregate claims process, respectively. 
Define the ruin probability by

$$
\psi(u)=\mathbb{P}\left(u+X_{t}<0 \text { for some } t>0\right) .
$$

In this paper, we are interested in estimating $\psi$ based on some observed values of $X$. We assume that the characteristic triple $\left(c, \sigma^{2}, \nu\right)$ is unknown. Note that if $c$ is the premium rate, it is known in practice, however, some additional unknown drift in the perturbation may be included in the total drift. If the Lévy density $\nu \equiv 0, X$ reduces to a Brownian motion with drift, and this leads to a parametric estimation problem. In this simple situation, we can use some traditional maximum likelihood methods to solve this problem. In the reminder of this paper, we shall not discuss this special case.

The reminder of this paper is organized as follows. In Section 2, we construct an estimator for $\psi$ via its Fourier transform. In Section 3, we study the consistency properties of our estimator. Some simulations are given in Section 4 to illustrate the performance of our estimator with finite sample size.

\section{The estimator}

Throughout this paper, we denote the Fourier transform of an integrable function $v$ by $\mathcal{F} v(s)=\int e^{i s x} v(x) d x$, where integral without indicated domain is taken over the whole real line. We use $\mathcal{R} s$ to denote the real part of a complex number $s$. We use $C, C^{\prime}, C^{\prime \prime}$ to denote positive generic constants that may vary at different steps. For two positive sequences $\left\{x_{n}\right\}_{n=1}^{\infty}$ and $\left\{y_{n}\right\}_{n=1}^{\infty}, x_{n} \lesssim y_{n}$ means that $x_{n} \leq C y_{n}$ for some constant $C$ for large index $n$.

\subsection{Fourier transform of $\psi$}

Let

$$
\mu_{k}=\int_{0}^{\infty} x^{k} \nu(d x), \quad k=1,2,3, \ldots,
$$

as long as the above integrals are finite. In particular, integration by parts gives

$$
\mu_{1}=\int_{0}^{\infty} \nu(x, \infty) d x<c
$$

thanks to the net profit condition (1.1). Let $\rho=\mu_{1} / c$. By Theorem 3.1 in Huzak et al. (2004) we have the following Pollaczek-Hinchin type formula for the survival probability,

$$
1-\psi(u)=(1-\rho) \sum_{j=0}^{\infty} \rho^{j}\left(G^{(j+1) *} * H^{j *}\right)(u), \quad u \geq 0,
$$


where $H(x)=\frac{1}{\mu_{1}} \int_{0}^{x} \nu(y, \infty) d y$, and $G$ is determined by the Laplace transform

$$
\int_{0}^{\infty} e^{-s x} d G(x)=\frac{c}{c+\frac{\sigma^{2}}{2} s} .
$$

Because ruin occurs immediately if the initial surplus is negative, we have $\psi(u)=1$ for $u<0$. Hence, $\psi$ is not integrable over the whole real line. To overcome this drawback, we modify the ruin probability by setting $\psi(u)=0$ for $u<0$. In the sequel, we only consider this modification and still denote it by $\psi$. It is not hard to see that $\psi$ is absolutely integrable after such modification. By formula (2.1) we obtain

$$
\begin{aligned}
\mathcal{F} \psi(s) & =\int_{0}^{\infty} e^{i s u} \psi(u) d u \\
& =(1-\rho) \sum_{j=0}^{\infty} \rho^{j} \frac{\left(\int_{0}^{\infty} e^{i s x} d G(x)\right)^{j+1}\left(\int_{0}^{\infty} e^{i s x} d H(x)\right)^{j}-1}{i s} \\
& =\frac{1}{i s}\left(\frac{(1-\rho) \int_{0}^{\infty} e^{i s x} d G(x)}{1-\rho \int_{0}^{\infty} e^{i s x} d G(x) \cdot \int_{0}^{\infty} e^{i s x} d H(x)}-1\right) .
\end{aligned}
$$

Note that

$$
\int_{0}^{\infty} e^{i s x} d G(x)=\frac{c}{c-\frac{\sigma^{2}}{2} i s}
$$

and by changing the order of integrals

$$
\int_{0}^{\infty} e^{i s x} d H(x)=\frac{1}{\mu_{1}} \int_{0}^{\infty} e^{i s x} \int_{x}^{\infty} \nu(d y) d x=\frac{1}{i s \mu_{1}} \int_{0}^{\infty}\left(e^{i s y}-1\right) \nu(d y) .
$$

Then we can write (2.2) in the following form,

$$
\mathcal{F} \psi(s)=\frac{\frac{\sigma^{2}}{2} i s+\frac{1}{i s} \int_{0}^{\infty}\left(e^{i s x}-1\right) \nu(d x)-\mu_{1}}{i c s+\frac{\sigma^{2}}{2} s^{2}-\int_{0}^{\infty}\left(e^{i s x}-1\right) \nu(d x)}=\frac{N(s)}{D(s)},
$$

where

$$
\begin{aligned}
& D(s)=-\Psi(-s)(i s)^{-1} \\
& N(s)=\frac{\sigma^{2}}{2}+\frac{1}{(i s)^{2}} \int_{0}^{\infty}\left(e^{i s x}-1-i s x\right) \nu(d x) .
\end{aligned}
$$

Lemma 1. Suppose that $\mu_{2}>0$. Then we have

$$
|\mathcal{F} \psi(s)| \leq \frac{C}{1 \vee|s|} .
$$


Proof. Note that

$$
\Psi(-s)=-i c s-\frac{\sigma^{2}}{2} s^{2}+\int_{0}^{\infty}(\cos (s x)-1) \nu(d x)+i \int_{0}^{\infty} \sin (s x) \nu(d x) .
$$

Using the inequality $\left|a_{1}+i a_{2}\right| \geq \max \left(\left|a_{1}\right|,\left|a_{2}\right|\right)$ for real numbers $a_{1}, a_{2}$, we obtain

$$
\begin{aligned}
|D(s)| & =\left|\Psi(-s) s^{-1}\right| \geq\left|c-\int_{0}^{\infty} \sin (s x) s^{-1} \nu(d x)\right| \\
& \geq c-\int_{0}^{\infty}\left|\frac{\sin (s x)}{s x}\right| x \nu(d x) \geq c-\mu_{1}>0,
\end{aligned}
$$

and

$$
|D(s)|=\left|\Psi(-s) s^{-1}\right| \geq \frac{\sigma^{2}}{2}|s|+\int_{0}^{\infty} \frac{1-\cos (s x)}{|s|} \nu(d x) \geq \frac{\sigma^{2}}{2}|s| .
$$

Hence, we have

$$
\frac{1}{|D(s)|} \leq C, \text { for } \sigma^{2}=0
$$

and

$$
\frac{1}{|D(s)|} \leq \frac{C}{1 \vee|s|}, \text { for } \sigma^{2}>0
$$

For $N(s)$, using the inequality

$$
\left|e^{i x}-1-i x\right| \leq \min \left(x^{2} / 2,2|x|\right),
$$

we have

$$
|N(s)| \leq \frac{\sigma^{2}}{2}+\min \left(\mu_{2} / 2,2 \mu_{1} /|s|\right)
$$

Finally, combining (2.4), (2.5) and (2.6) we complete the proof.

Remark 1. Suppose that $\mu_{2}<\infty$. Using the inequality $\left|a_{1}+i a_{2}\right| \leq\left|a_{1}\right|+\left|a_{2}\right|$ we obtain

$$
\begin{aligned}
|D(s)| & \leq c+\int_{0}^{\infty}\left|\frac{\sin (s x)}{s x}\right| x \nu(d x)+\frac{\sigma^{2}}{2}|s|+\int_{0}^{\infty} \frac{1-\cos (s x)}{|s|} \nu(d x) \\
& \leq c+\mu_{1}+\frac{\sigma^{2}+\mu_{2}}{2}|s|,
\end{aligned}
$$

where we have used the inequality $1-\cos x \leq \frac{x^{2}}{2}$ in the second step.

It follows from Lemma 1 that $\mathcal{F} \psi$ is not absolutely integrable but square integrable. Since $\psi(u)$ is a monotonic function, using "principal value" integral we can still recover $\psi$ by Fourier inversion, i.e.

$$
\psi(u)=\frac{1}{2 \pi} P V \int e^{-i s u} \frac{N(s)}{D(s)} d s=\frac{1}{2 \pi} \lim _{M \rightarrow \infty} \int_{-M}^{M} e^{-i s u} \frac{N(s)}{D(s)} d s .
$$




\subsection{Constructing an estimator}

Recently, a lot of contributions have been made to the estimation of the Lévy characteristic triplet $\left(c, \sigma^{2}, \nu\right)$. See, for example, Comte and Genon-Catalot $(2009,2010)$, Gugushvili (2009, 2012), Kappus and Reiß(2010), Kappus (2014). Once we can get some estimates of the Lévy triplet, we can use (2.1) to estimate the survival probability by a plug-in technique. However, due to the complexity of (2.1), such straightforward method is not applicable in practical applications. It follows from (2.3) that the Fourier transform of the ruin probability is closely related to the characteristic exponent $\Psi$, and we can recover $\Psi$ from the characteristic function $\phi_{X_{t}}$. Hence, it is more convenient to use characteristic function and Fourier transform to construct the estimator.

Assume that some discrete observed values of $X$, i.e. $\left\{X_{k \Delta}, k=0,1,2 \ldots, n\right\}$, are available, where $\Delta>0$ is the sampling interval. Different from Zhang and Yang (2013), we assume that $\Delta$ is fixed. Let

$$
Z_{k}=Z_{k}^{\Delta}=X_{k \Delta}-X_{(k-1) \Delta}, \quad k=1,2, \ldots, n .
$$

Let $Z$ be a generic version of $Z_{k}$ and denote the characteristic function of $Z$ by $\phi_{Z}$. Then we have

$$
\phi_{Z}(s)=e^{\Delta \Psi(s)} .
$$

In order to estimate the ruin probability, we firstly estimate its Fourier transform. It follows from (2.3) that we need to estimate the following quantities,

$$
D(s), \quad N(s) \text {. }
$$

First, we estimate $D(s)$. Note that $D(s)=-\frac{1}{\Delta}(i s)^{-1} \log \phi_{Z}(-s)$, where Log denotes the distinguished logarithm (see e.g. Theorem 7.6.2. in Chung (2001)). Let $\widehat{\phi}_{Z}(s)=$ $\frac{1}{n} \sum_{j=1}^{n} e^{i s Z_{j}}$ be the empirical characteristic function, and set

$$
\widetilde{\phi}_{Z}(s)=\widehat{\phi}_{Z}(s) \mathbf{1}_{A_{n, s}}+\mathbf{1}_{A_{n, s}^{c}},
$$

where $A_{n, s}=\left\{\left|\widehat{\phi}_{Z}(s)\right| \geq n^{-\frac{1}{2}}\right\}$. Then $\widetilde{\phi}_{Z}(s)$ never vanishes, although it is not continuous. Let $\left\{r_{n}\right\}$ be a sequence such that $r_{n}>0$ and $r_{n} \rightarrow 0$. We estimate $D(s)$ by

$$
\widehat{D}(s)=\widetilde{D}(s) \mathbf{1}_{B_{n, s}}+r_{n} \mathbf{1}_{B_{n, s}^{c}},
$$

where

$$
\widetilde{D}(s)=-\frac{1}{\Delta}(i s)^{-1} \log \widetilde{\phi}_{Z}(-s), \quad B_{n, s}=\left\{|\widetilde{D}(s)| \geq r_{n}\right\} .
$$

Next, we estimate $N(s)$. It is readily seen that

$$
N(s)=(i s)^{-1}\left(c-\mu_{1}-D(s)\right) .
$$


For the mean value $c-\mu_{1}$, it can be estimated by

$$
\frac{1}{n \Delta} \sum_{j=1}^{n} Z_{j}
$$

By (2.10), we can estimate $N(s)$ by

$$
\widehat{N}(s):=\frac{1}{i s}\left(\frac{1}{n \Delta} \sum_{j=1}^{n} Z_{j}-\widehat{D}(s)\right) .
$$

Finally, by (2.8), (2.9) and (2.11), we propose the following estimator for ruin probability,

$$
\widehat{\psi}(u)=\frac{1}{2 \pi} \mathcal{R} \int_{m_{n}}^{M_{n}} e^{-i u s} \frac{\widehat{N}(s)}{\widehat{D}(s)} d s+\frac{1}{2 \pi} \mathcal{R} \int_{-M_{n}}^{-m_{n}} e^{-i u s} \frac{\widehat{N}(s)}{\widehat{D}(s)} d s, \quad u>0,
$$

where $m_{n}$ and $M_{n}$ are positive cut-off numbers such that $m_{n} \rightarrow 0$ and $M_{n} \rightarrow \infty$ as $n \rightarrow \infty$.

\section{Conditions and convergence rates}

In this section, we study the consistency properties of the estimator. It is known that the convergence rate of the Lévy characteristic triplet depends heavily on the decay rate of the characteristic function $\phi_{Z}$. See, for example, Kappus and Rieß(2010), Gugushvili (2012) and Kappus (2014). In order to present the main result of this paper, it is more convenient to classify the characteristic function $\phi_{Z}$ according to its decay rate.

We consider the following three classes of characteristic functions.

- Class I (bounded away from zero)

$$
\begin{aligned}
\Sigma_{1}\left(d_{0}, d_{1}\right)=\{\phi: & \phi \text { is a characteristic function, } \\
& \left.d_{0} \leq|\phi(s)| \leq d_{1} \text { uniformly in } s \in \mathbb{R}, d_{0}, d_{1}>0\right\} .
\end{aligned}
$$

- Class II (polynomial decay rate)

$$
\begin{aligned}
\Sigma_{2}\left(d_{0}, d_{1}, \beta\right)=\{ & \phi: \phi \text { is a characteristic function, } \\
& \frac{d_{0}}{\left(1+s^{2}\right)^{\beta / 2}} \leq|\phi(s)| \leq \frac{d_{1}}{\left(1+s^{2}\right)^{\beta / 2}} \text { uniformly in } s \in \mathbb{R}, \\
& \left.d_{0}, d_{1}, \beta>0\right\} .
\end{aligned}
$$


- Class III (exponent decay rate)

$$
\Sigma_{3}\left(d_{0}, d_{1}, \beta_{0}, \beta_{1}, \beta, \gamma_{0}, \gamma_{1}\right)
$$

$=\{\phi: \phi$ is a characteristic function,

$$
\begin{aligned}
& \frac{d_{0} e^{-\gamma_{0}|s|^{\beta}}}{\left(1+s^{2}\right)^{\beta_{0} / 2}} \leq|\phi(s)| \leq \frac{d_{1} e^{-\gamma_{1}|s|^{\beta}}}{\left(1+s^{2}\right)^{\beta_{1} / 2}} \text { uniformly in } s \in \mathbb{R}, \\
& \left.d_{0}, d_{1}, \beta, \gamma_{0}, \gamma_{1}>0, \beta_{0}, \beta_{1} \in \mathbb{R}\right\} .
\end{aligned}
$$

Remark 2. If the characteristic function $\phi_{Z}$ decays at polynomial rate, then the corresponding density function is ordinary smooth; if the characteristic function $\phi_{Z}$ decays at exponential rate, then the corresponding density function is supersmooth. See, for example, Fan (1991).

We give some examples.

Example 1 (Drift-Compound Poisson process). Let $X_{t}=c t-S_{t}$, where $S_{t}$ is a compound Poisson process. Then

$$
\Psi(s)=i c s+\int_{0}^{\infty}\left(e^{-i s x}-1\right) \nu(d x)
$$

with $\int_{0}^{\infty} \nu(d x)<\infty$. Note that $\left|\phi_{Z}(s)\right|=e^{\Delta \mathcal{R} \Psi(s)}$ and $\mathcal{R} \Psi(s)=\int_{0}^{\infty}(\cos (s x)-1) \nu(d x)$. This leads to

$$
e^{-2 \Delta \int_{0}^{\infty} \nu(d x)} \leq\left|\phi_{Z}(s)\right| \leq 1 .
$$

Then the characteristic function $\phi_{Z}$ belongs to class $I$.

Example 2 (Drift-Lévy-Gamma process). Let $X_{t}=c t-\Gamma_{t}$, where for fixed $t, \Gamma_{t}$ follows Gamma distribution with parameters $\left(\tau_{0} t, \tau_{1}\right)$ with $\tau_{0}, \tau_{1}>0$. The characteristic function of $Z$ is given by

$$
\phi_{Z}(s)=e^{i \Delta c s}\left(\frac{\tau_{1}}{\tau_{1}+i s}\right)^{\tau_{0} \Delta} .
$$

Hence, the characteristic function $\phi_{Z}$ belongs to class II.

Example 3 (Drift-Inverse Gauss process). Let $X_{t}=c t-I G_{t}$, where $I G_{t}$ is an inverse Gauss process with Lévy measure

$$
\nu(d x)=\frac{1}{\sqrt{2 \pi x^{3}}} e^{-\frac{b^{2}}{2} x} \mathbf{1}_{(x>0)} d x, \quad b>0 .
$$

Then for some real number $c^{\prime}$ we have

$$
\Psi(s)=i c^{\prime} s+b-\sqrt{b^{2}-2 i s} .
$$

which implies that the characteristic function $\phi_{Z}$ belongs to class III. 
Example 4 (Drift+Brownian motion-subordinator). In this case, we have

$$
\Psi(s)=i c s-\frac{\sigma^{2}}{2} s^{2}-\int_{0}^{\infty}\left(e^{-i s x}-1\right) \nu(d x), \quad \sigma^{2}>0 .
$$

Obviously, in this example the characteristic function $\phi_{Z}$ belongs to class III.

The convergence rate of the estimator also depends on the choice of the parameters $r_{n}, m_{n}$ and $M_{n}$. We consider the following three conditions, which correspond to the above three classes of characteristic functions.

\section{Condition M}

(M.1) $r_{n}=n^{-\kappa_{1}}, m_{n}=n^{-\kappa_{2}}$, where $\kappa_{1}, \kappa_{2}>0$ and $1-2\left(\kappa_{1}+\kappa_{2}\right)>0$.

(M.2) $r_{n}=n^{-\kappa_{1}}, m_{n}=n^{-\kappa_{2}}, M_{n}=n^{\alpha}$, where $\kappa_{1}, \kappa_{2}, \alpha, \beta>0$, and $1-2\left(\kappa_{1}+\kappa_{2}+\alpha \beta\right)>0$.

(M.3) $r_{n}=n^{-\kappa_{1}}, m_{n}=n^{-\kappa_{2}}, M_{n}=(\alpha \log n)^{\frac{1}{\beta}}$, where $\kappa_{1}, \kappa_{2}, \alpha, \gamma_{0}>0,1-2\left(\kappa_{1}+\kappa_{2}+\right.$ $\left.\gamma_{0} \alpha\right)>0$.

Now we present two lemmas that are useful to prove the consistency of our estimator.

Lemma 2. (1) If $\phi_{Z} \in \Sigma_{1}\left(d_{0}, d_{1}\right)$, then

$$
\sup _{s \in(-\infty,+\infty)} \mathbb{P}\left(A_{n, s}^{c}\right) \lesssim \exp \left(-\frac{3 d_{0}^{2}}{8\left(d_{0}+3\right)} n\right) .
$$

(2) If $\phi_{Z} \in \Sigma_{2}\left(d_{0}, d_{1}, \beta\right), M_{n}=n^{\alpha}$ and $1-2 \alpha \beta>0$, then

$$
\sup _{|s| \leq \mid M_{n}} \mathbb{P}\left(A_{n, s}^{c}\right) \lesssim \exp \left(-\frac{d_{0}^{2}}{16} n^{1-2 \alpha \beta}\right) .
$$

(3) If $\phi_{Z} \in \Sigma_{3}\left(d_{0}, d_{1}, \beta_{0}, \beta_{1}, \beta, \gamma_{0}, \gamma_{1}\right), M_{n}=(\alpha \log n)^{\frac{1}{\beta}}$ and $1-2 \alpha \gamma_{0}>0$, then for $\alpha<\alpha^{\prime}<\frac{1}{2 \gamma_{0}}$ we have

$$
\sup _{|s| \leq M_{n}} \mathbb{P}\left(A_{n, s}^{c}\right) \lesssim \exp \left(-\frac{d_{0}^{2}}{16} n^{1-2 \gamma_{0} \alpha^{\prime}}\right)
$$

Proof. Using the inequality $|a+b| \geq|a|-|b|$ we have

$$
\mathbb{P}\left(\left|\widehat{\phi}_{Z}(s)\right|<n^{-\frac{1}{2}}\right) \leq \mathbb{P}\left(\left|\widehat{\phi}_{Z}(s)-\phi_{Z}(s)\right| \geq\left|\phi_{Z}(s)\right|-n^{-\frac{1}{2}}\right)
$$




$$
=\mathbb{P}\left(\left|\sum_{j=1}^{n}\left(e^{i s Z_{j}}-\mathbb{E} e^{i s Z_{j}}\right)\right| \geq n\left|\phi_{Z}(s)\right|-n^{\frac{1}{2}}\right) .
$$

We apply Bernstein's inequality ( see Appendix A) to bound the probability on the right hand side of (3.4). Note that $e^{i s Z_{1}}-\mathbb{E} e^{i s Z_{1}}, \ldots, e^{i s Z_{n}}-\mathbb{E} e^{i s Z_{n}}$ are independent centered random variables and satisfy

$$
\left|e^{i s Z_{j}}-\mathbb{E} e^{i s Z_{j}}\right| \leq 2, \quad \sum_{j=1}^{n} \operatorname{Var}\left(e^{i s Z_{j}}-\mathbb{E} e^{i s Z_{j}}\right) \leq n .
$$

If $\phi_{Z} \in \Sigma_{1}\left(d_{0}, d_{1}\right)$, then for large enough $n$ we have

$$
n\left|\phi_{Z}(s)\right|-n^{\frac{1}{2}} \geq \frac{1}{2} d_{0} n
$$

uniformly in $s$. Hence, by Bernstein's inequality and (3.4) we obtain

$$
\mathbb{P}\left(A_{n, s}^{c}\right) \leq C \cdot \mathbb{P}\left(\mid \sum_{j=1}^{n}\left(e^{i s Z_{j}}-\mathbb{E} e^{i s Z_{j}} \mid \geq \frac{1}{2} d_{0} n\right) \leq C \cdot \exp \left(-\frac{3 d_{0}^{2}}{8\left(d_{0}+3\right)} n\right) .\right.
$$

If $\phi_{Z} \in \Sigma_{1}\left(d_{0}, d_{1}, \beta\right)$, then for large enough $n$ and $|s| \leq M_{n}=n^{\alpha}$ we have

$$
n\left|\phi_{Z}(s)\right|-n^{\frac{1}{2}} \geq d_{0} n\left(1+M_{n}^{2}\right)^{-\frac{\beta}{2}}-n^{\frac{1}{2}} \geq d_{0} n^{1-\alpha \beta}-n^{\frac{1}{2}} \geq \frac{1}{2} d_{0} n^{1-\alpha \beta} .
$$

By Bernstein's inequality and (3.4) we have

$$
\begin{aligned}
\mathbb{P}\left(A_{n, s}^{c}\right) & \lesssim \mathbb{P}\left(\mid \sum_{j=1}^{n}\left(e^{i s Z_{j}}-\mathbb{E} e^{i s Z_{j}} \mid \geq \frac{1}{2} d_{0} n^{1-\alpha \beta}\right)\right. \\
& \lesssim \exp \left(-\frac{\frac{1}{8} d_{0}^{2} n^{2-2 \alpha \beta}}{n+\frac{1}{3} d_{0} n^{1-\alpha \beta}}\right) \\
& \lesssim \exp \left(-\frac{1}{16} d_{0}^{2} n^{1-2 \alpha \beta}\right) .
\end{aligned}
$$

If $\phi_{Z} \in \Sigma_{3}\left(d_{0}, d_{1}, \beta_{0}, \beta_{1}, \beta, \gamma_{0}, \gamma_{1}\right)$, then for $|s| \leq(\alpha \log n)^{\frac{1}{\beta}}$ we have

$$
\begin{aligned}
n\left|\phi_{Z}(s)\right|-n^{\frac{1}{2}} & \geq d_{0} n\left(1+s^{2}\right)^{-\beta_{0} / 2} \exp \left(-\gamma_{0}|s|^{\beta}\right)-n^{\frac{1}{2}} \\
& \geq d_{0}\left(1+s^{2}\right)^{-\beta_{0} / 2} n^{1-\gamma_{0} \alpha}-n^{\frac{1}{2}} \\
& \geq \frac{1}{2} d_{0} n^{1-\gamma_{0} \alpha^{\prime}} \quad(\text { for large enough } n) .
\end{aligned}
$$


Again, by Bernstein's inequality and (3.4) we obtain

$$
\mathbb{P}\left(A_{n, s}^{c}\right) \lesssim \mathbb{P}\left(\mid \sum_{j=1}^{n}\left(e^{i s Z_{j}}-\mathbb{E} e^{i s Z_{j}} \mid \geq \frac{1}{2} d_{0} n^{1-\gamma_{0} \alpha^{\prime}}\right) \lesssim \exp \left(-\frac{1}{16} d_{0}^{2} n^{1-2 \gamma_{0} \alpha^{\prime}}\right) .\right.
$$

Then (3.1)-(3.3) follow immediately from the above results.

Lemma 3. (1) If $\phi_{Z}(s) \in \Sigma_{1}\left(d_{0}, d_{1}\right)$, then under condition (M.1) we have

$$
\sup _{m_{n} \leq|s| \leq M_{n}} \mathbb{P}\left(\mid B_{n, s}^{c}\right) \lesssim \exp \left(-\frac{1}{16} d_{0}^{2} \Delta^{2} n^{1-2\left(\kappa_{1}+\kappa_{2}\right)}\right) .
$$

(2) If $\phi_{Z}(s) \in \Sigma_{2}\left(d_{0}, d_{1}, \beta\right)$, then under condition (M.2) we have

$$
\sup _{m_{n} \leq|s| \leq \mid M_{n}} \mathbb{P}\left(B_{n, s}^{c}\right) \lesssim \exp \left(-\frac{1}{64} d_{0}^{2} \Delta^{2} n^{1-2\left(\kappa_{1}+\kappa_{2}\right)-2 \alpha \beta}\right) .
$$

(3) If $\phi_{Z} \in \Sigma_{3}\left(d_{0}, d_{1}, \beta_{0}, \beta_{1}, \beta, \gamma_{0}, \gamma_{1}\right)$, then under condition (M.3), we have for $\alpha<$ $\alpha^{\prime}<\frac{1-2\left(\kappa_{1}+\kappa_{2}\right)}{2 \gamma_{0}}$

$$
\sup _{m_{n} \leq|s| \leq M_{n}} \mathbb{P}\left(B_{n, s}^{c}\right) \lesssim \exp \left(-\frac{1}{64} d_{0}^{2} \Delta^{2} n^{1-2\left(\kappa_{1}+\kappa_{2}\right)-2 \gamma_{0} \alpha^{\prime}}\right) .
$$

Proof. It follows from (2.4) and (2.5) that $|D(s)|$ is bounded away from zero, which implies that for sufficiently large $n$,

$$
|D(s)| \geq 2 r_{n}
$$

uniformly in $s$, since $r_{n} \rightarrow 0$. Hence, for $|s| \in\left[m_{n}, M_{n}\right]$ we have

$$
\begin{aligned}
\mathbb{P}\left(|\widetilde{D}(s)|<r_{n}\right) & \leq \mathbb{P}\left(|\widetilde{D}(s)-D(s)|>|D(s)|-r_{n}\right) \\
& \lesssim \mathbb{P}\left(|\widetilde{D}(s)-D(s)|>r_{n}\right) \\
& \lesssim \mathbb{P}\left(\left\{|\widetilde{D}(s)-D(s)|>r_{n}\right\} \cap A_{n, s}\right)+\mathbb{P}\left(A_{n, s}^{c}\right) \\
& \lesssim \mathbb{P}\left(|\log (1+\Lambda(s))|>\Delta m_{n} r_{n}\right)+\mathbb{P}\left(A_{n, s}^{c}\right)
\end{aligned}
$$

where $\Lambda(s)=\widehat{\phi}_{Z}(-s) / \phi_{Z}(-s)-1$.

Upper bounds for $\mathbb{P}\left(A_{n, s}^{c}\right)$ have been given in Lemma 2 . Hence, we only need to bound the first probability on the right hand side of (3.8). Note that $\Delta m_{n} r_{n} \rightarrow 0$. Using the inequality

$$
|\log (1+z)| \leq 2|z|, \quad \text { for }|z| \leq \frac{1}{2},
$$


we have

$$
\begin{aligned}
& \mathbb{P}\left(|\log (1+\Lambda(s))|>\Delta m_{n} r_{n}\right) \\
\leq & \mathbb{P}\left(|\log (1+\Lambda(s))| \geq \Delta m_{n} r_{n},|\Lambda(s)|<\frac{1}{2} \Delta m_{n} r_{n}\right)+\mathbb{P}\left(|\Lambda(s)| \geq \frac{1}{2} \Delta m_{n} r_{n}\right) \\
\lesssim & \mathbb{P}\left(|\Lambda(s)| \geq \frac{1}{2} \Delta m_{n} r_{n}\right) \\
= & \mathbb{P}\left(\left|\widehat{\phi}_{Z}(-s)-\phi_{Z}(-s)\right| \geq \frac{1}{2} \Delta m_{n} r_{n}\left|\phi_{Z}(-s)\right|\right) \\
= & \mathbb{P}\left(\left|\sum_{j=1}^{n}\left(e^{-i s Z_{j}}-\mathbb{E} e^{-i s Z_{j}}\right)\right| \geq \frac{1}{2} \Delta n m_{n} r_{n}\left|\phi_{Z}(-s)\right|\right) .
\end{aligned}
$$

We will use Bernstein's inequality to bound the above probability.

If $\phi_{Z} \in \Sigma_{1}\left(d_{0}, d_{1}\right)$, then under condition (M.1) we have

$$
\frac{1}{2} \Delta n m_{n} r_{n}\left|\phi_{Z}(-s)\right| \geq \frac{1}{2} d_{0} \Delta n^{1-\kappa_{1}-\kappa_{2}} .
$$

Applying Bernstein's inequality to (3.10) gives

$$
\begin{aligned}
\mathbb{P}\left(|\log (1+\Lambda(s))|>\Delta m_{n} r_{n}\right) & \lesssim \mathbb{P}\left(\left|\sum_{j=1}^{n}\left(e^{-i s Z_{j}}-\mathbb{E} e^{-i s Z_{j}}\right)\right| \geq \frac{1}{2} d_{0} \Delta n^{1-\kappa_{1}-\kappa_{2}}\right) \\
& \lesssim \exp \left(-\frac{1}{16} d_{0}^{2} \Delta^{2} n^{1-2\left(\kappa_{1}+\kappa_{2}\right)}\right),
\end{aligned}
$$

which, together with (3.1) and (3.8), gives (3.5).

If $\phi_{Z} \in \Sigma_{2}\left(d_{0}, d_{1}, \beta\right)$, then under condition (M.2), for $|s| \leq n^{\alpha}$ and large enough $n$ we have

$$
\frac{1}{2} \Delta n m_{n} r_{n}\left|\phi_{Z}(-s)\right| \geq \frac{1}{2} d_{0} \Delta n m_{n} r_{n}\left(1+s^{2}\right)^{-\frac{\beta}{2}} \geq \frac{1}{4} d_{0} \Delta n^{1-\kappa_{1}-\kappa_{2}-\alpha \beta} .
$$

Hence, by (3.10) and Bernstein's inequality we obtain

$$
\begin{aligned}
& \mathbb{P}\left(|\log (1+\Lambda(s))|>\Delta m_{n} r_{n}\right) \\
\lesssim & \mathbb{P}\left(\left|\sum_{j=1}^{n}\left(e^{-i s Z_{j}}-\mathbb{E} e^{-i s Z_{j}}\right)\right| \geq \frac{1}{4} d_{0} \Delta n^{1-\kappa_{1}-\kappa_{2}-\alpha \beta}\right) \\
\lesssim & \exp \left(-\frac{1}{64} d_{0}^{2} \Delta^{2} n^{1-2\left(\kappa_{1}+\kappa_{2}+\alpha \beta\right)}\right),
\end{aligned}
$$

which, together with (3.2) and (3.8), gives (3.6). 
If $\phi_{Z} \in \Sigma_{3}\left(d_{0}, d_{1}, \beta_{0}, \beta_{1}, \beta, \gamma_{0}, \gamma_{1}\right)$, then under condition (M.3), for $|s| \leq(\alpha \log n)^{\frac{1}{\beta}}$, $\alpha<\alpha^{\prime}<\frac{1-2\left(\kappa_{1}+\kappa_{2}\right)}{2 \gamma_{0}}$ and large enough $n$, we have

$$
\frac{1}{2} \Delta n m_{n} r_{n}\left|\phi_{Z}(-s)\right| \geq \frac{1}{2} d_{0} \Delta n m_{n} r_{n}\left(1+s^{2}\right)^{-\frac{\beta}{2}} \exp \left(-\gamma_{0}|s|^{\beta}\right) \geq \frac{1}{4} d_{0} \Delta n^{1-\kappa_{1}-\kappa_{2}-\gamma_{0} \alpha^{\prime}} .
$$

Again, we can apply Berstein's inequality to (3.10) to obtain

$$
\begin{aligned}
& \mathbb{P}\left(|\log (1+\Lambda(s))|>\Delta m_{n} r_{n}\right) \\
\lesssim & \mathbb{P}\left(\left|\sum_{j=1}^{n}\left(e^{-i s Z_{j}}-\mathbb{E} e^{-i s Z_{j}}\right)\right| \geq \frac{1}{4} d_{0} \Delta n^{1-\kappa_{1}-\kappa_{2}-\gamma_{0} \alpha^{\prime}}\right) \\
\lesssim & \exp \left(-\frac{1}{64} d_{0}^{2} \Delta^{2} n^{1-2\left(\kappa_{1}+\kappa_{2}+\gamma_{0} \alpha^{\prime}\right)}\right),
\end{aligned}
$$

which, together with (3.3) and (3.8), gives (3.7).

The following two propositions are also useful.

Proposition 1. Let $p \geq 2$ be an integer.

(1) If $\phi_{Z} \in \Sigma_{1}\left(d_{0}, d_{1}\right)$, then under condition (M.1) we have

$$
\sup _{|s| \in\left[m_{n}, M_{n}\right]} \mathbb{E}|\widehat{D}(s)-D(s)|^{p} \lesssim n^{-\frac{p}{2}+p \kappa_{2}},
$$

and for $|s| \in\left[m_{n}, M_{n}\right]$

$$
\mathbb{E}\left|\frac{1}{\widehat{D}(s)}-\frac{1}{D(s)}\right|^{p} \lesssim \frac{1}{|D(s)|^{p}} n^{-\frac{p}{2}+p\left(\kappa_{1}+\kappa_{2}\right)} .
$$

(2) If $\phi_{Z} \in \Sigma_{2}\left(d_{0}, d_{1}, \beta\right)$, then under condition (M.2) we have

$$
\sup _{|s| \in\left[m_{n}, M_{n}\right]} \mathbb{E}|\widehat{D}(s)-D(s)|^{p} \lesssim n^{-\frac{p}{2}+p \theta},
$$

and for $|s| \in\left[m_{n}, M_{n}\right]$

$$
\mathbb{E}\left|\frac{1}{\widehat{D}(s)}-\frac{1}{D(s)}\right|^{p} \lesssim \frac{1}{|D(s)|^{p}} n^{-\frac{p}{2}+p\left(\kappa_{1}+\theta\right)},
$$

where $\theta=\max \left(\kappa_{2}, \alpha(\beta-1)\right)$. 
(3) If $\phi_{Z} \in \Sigma_{3}\left(d_{0}, d_{1}, \beta_{0}, \beta_{1}, \beta, \gamma_{0}, \gamma_{1}\right)$, then under condition (M.3) we have

$$
\begin{aligned}
& \sup _{|s| \in\left[m_{n}, M_{n}\right]} \mathbb{E}|\widehat{D}(s)-D(s)|^{p} \lesssim \max \left(n^{-\frac{p}{2}+p \kappa_{2}},(\alpha \log n)^{\frac{p\left(\beta_{0}-1\right)}{\beta}} n^{-\frac{p}{2}+p \gamma_{0} \alpha}\right) . \\
& \text { and for }|s| \in\left[m_{n}, M_{n}\right] \\
& \mathbb{E}\left|\frac{1}{\widehat{D}(s)}-\frac{1}{D(s)}\right|^{p} \lesssim \frac{1}{|D(s)|^{p}} \max \left(n^{-\frac{p}{2}+p\left(\kappa_{1}+\kappa_{2}\right)},(\alpha \log n)^{\frac{p\left(\beta_{0}-1\right)}{\beta}} n^{-\frac{p}{2}+p\left(\kappa_{1}+\gamma_{0} \alpha\right)}\right) .
\end{aligned}
$$

Proof. Recall the definitions of $A_{n, s}$ and $B_{n, s}$. We note that $\widetilde{D}(s)=0$ on the complement set $A_{n,-s}^{c}$. Hence, $B_{n, s} \cap A_{n,-s}^{c}$ is an empty set. Then for $|s| \in\left[m_{n}, M_{n}\right]$, we have

$$
\begin{aligned}
& \mathbb{E}|\widehat{D}(s)-D(s)|^{p} \\
= & \mathbb{E}\left(|\widetilde{D}(s)-D(s)|^{p} \mathbf{1}_{B_{n, s}}\right)+\mathbb{E}\left(\left|r_{n}-D(s)\right|^{p} \mathbf{1}_{B_{n, s}^{c}}\right) \\
= & \mathbb{E}\left(|\widetilde{D}(s)-D(s)|^{p} \mathbf{1}_{B_{n, s} \cap A_{n,-s} \cap\left\{|\Lambda(s)| \leq \frac{1}{2}\right\}}\right) \\
& +\mathbb{E}\left(|\widetilde{D}(s)-D(s)|^{p} \mathbf{1}_{B_{n, s} \cap A_{n,-s} \cap\left\{|\Lambda(s)|>\frac{1}{2}\right\}}\right)+\mathbb{E}\left(\left|r_{n}-D(s)\right|^{p} \mathbf{1}_{B_{n, s}^{c}}\right) \\
:= & I_{1}+I_{2}+I_{3},
\end{aligned}
$$

where $\Lambda(s)=\widehat{\phi}_{Z}(-s) / \phi_{Z}(-s)-1$.

For $I_{3}$, by $C_{r}$ inequality and Remark 1 we have

$$
I_{3} \lesssim\left(r_{n}^{p}+M_{n}^{p}\right) \mathbb{P}\left(B_{n, s}^{c}\right)
$$

for $|s| \leq M_{n}$. Furthermore, by the exponential bounds for $\mathbb{P}\left(B_{n, s}^{c}\right)$ given in Lemma 3, we have

$$
\sup _{|s| \in\left[m_{n}, M_{n}\right]} I_{3} \lesssim\left(r_{n}^{p}+M_{n}^{p}\right) \exp \left(-C^{\prime} n^{\epsilon}\right)
$$

for some $C^{\prime}>0$ and $0<\epsilon<1$.

Next, we derive upper bounds for $I_{2}$. On $B_{n, s} \cap A_{n,-s}$ we have

$$
|\widetilde{D}(s)| \lesssim|s|^{-1} \log n .
$$

Hence, using $C_{r}$ inequality and Remark 1 , for $|s| \in\left[m_{n}, M_{n}\right]$ we have

$$
\begin{aligned}
I_{2} & \lesssim\left(m_{n}^{-p}(\log n)^{p}+M_{n}^{p}\right) \mathbb{P}(|\Lambda(s)|>1 / 2) \\
& \lesssim\left(m_{n}^{-p}(\log n)^{p}+M_{n}^{p}\right) \mathbb{P}\left(|\Lambda(s)|>\frac{1}{2} \Delta m_{n} r_{n}\right),
\end{aligned}
$$


where the second step follows from the fact that $m_{n} r_{n} \rightarrow 0$ as $n \rightarrow \infty$. By the above inequality and the proof of Lemma 3 we know that

$$
\sup _{|s| \in\left[m_{n}, M_{n}\right]} I_{2} \lesssim\left(m_{n}^{-p}(\log n)^{p}+M_{n}^{p}\right) \exp \left(-C^{\prime \prime} n^{\epsilon^{\prime}}\right)
$$

for some $C^{\prime \prime}>0$ and $0<\epsilon^{\prime}<1$.

For $I_{1}$, using (3.9) and the Rosenthal inequality (see Appendix A) we can obtain

$$
\begin{aligned}
I_{1} & =\frac{1}{(\Delta|s|)^{p}} \mathbb{E}\left(|\log (1+\Lambda(s))|^{p} \mathbf{1}_{B_{n, s} \cap A_{n,-s} \cap\left\{|\Lambda(s)| \leq \frac{1}{2}\right\}}\right) \\
& \lesssim \frac{1}{\left|s \phi_{Z}(-s)\right|^{p}} \mathbb{E}\left|\widehat{\phi}_{Z}(-s)-\phi_{Z}(-s)\right|^{p} \\
& \lesssim \frac{1}{\left|s \phi_{Z}(s)\right|^{p}}\left(\frac{1}{n^{p-1}}+\frac{1}{n^{p / 2}}\right) \\
& \lesssim \frac{n^{-p / 2}}{\left|s \phi_{Z}(s)\right|^{p}} \quad(\text { for } p \geq 2) .
\end{aligned}
$$

Hence, in order to analyze the upper bound in (3.15), we need to distinguish the decay rates of $\phi_{Z}$. We consider three cases.

(1) If $\phi_{Z} \in \Sigma_{1}\left(d_{0}, d_{1}\right)$, then under condition (M.1), we obtain from (3.15) that

$$
\sup _{|s| \in\left[m_{n}, M_{n}\right]} I_{1} \lesssim n^{-\frac{p}{2}+p \kappa_{2}} .
$$

(2) If $\phi_{Z} \in \Sigma_{2}\left(d_{0}, d_{1}, \beta\right)$, we have

$$
\left|s \phi_{Z}(s)\right|^{-p} \leq d_{0}^{-p}\left(1+s^{2}\right)^{\frac{p \beta}{2}}|s|^{-p} \lesssim\left\{\begin{array}{l}
|s|^{-p},|s| \leq 1, \\
|s|^{p(\beta-1)},|s|>1 .
\end{array}\right.
$$

Then under condition (M.2), (3.15) yields

$$
\sup _{|s| \in\left[m_{n}, M_{n}\right]} I_{1} \lesssim \max \left(n^{-\frac{p}{2}+p \kappa_{2}}, n^{-\frac{p}{2}+p \alpha(\beta-1)}\right)=n^{-\frac{p}{2}+p \theta} .
$$

(3) If $\phi_{Z} \in \Sigma_{3}\left(d_{0}, d_{1}, \beta_{0}, \beta_{1}, \beta, \gamma_{0}, \gamma_{1}\right)$, we have

$$
\begin{aligned}
\left|s \phi_{Z}(s)\right|^{-p} & \leq d_{0}^{-p}|s|^{-p}\left(1+s^{2}\right)^{\frac{p \beta_{0}}{2}} \exp \left(p \gamma_{0}|s|^{\beta}\right) \\
& \lesssim\left\{\begin{array}{l}
|s|^{-p}, \quad|s| \leq 1, \\
|s|^{p\left(\beta_{0}-1\right)} \exp \left(p \gamma_{0}|s|^{\beta}\right), \quad|s|>1 .
\end{array}\right.
\end{aligned}
$$


Then

$$
\sup _{|s| \in\left[m_{n}, M_{n}\right]}\left|s \phi_{Z}(s)\right| \lesssim \max \left(n^{p \kappa_{2}},(\alpha \log n)^{\frac{p\left(\beta_{0}-1\right)}{\beta}} n^{p \gamma_{0} \alpha}\right) .
$$

By (3.15) we obtain

$$
\sup _{|s| \in\left[m_{n}, M_{n}\right]} I_{1} \lesssim \max \left(n^{-\frac{p}{2}+p \kappa_{2}},(\alpha \log n)^{\frac{p\left(\beta_{0}-1\right)}{\beta}} n^{-\frac{p}{2}+p \gamma_{0} \alpha}\right) .
$$

Compared with (3.16)-(3.18), the upper bounds obtained in (3.13) and (3.14) are negligible. Then the upper bounds for $\mathbb{E}|\widehat{D}(s)-D(s)|^{p}$ are obtained.

Finally, from the definition of $\widehat{D}(s)$ we know

$$
\mathbb{E}\left|\frac{1}{\widehat{D}(s)}-\frac{1}{D(s)}\right|^{p} \leq \frac{r_{n}^{-p}}{|D(s)|^{p}} \mathbb{E}|\widehat{D}(s)-D(s)|^{p} .
$$

Then the reminder of the proof follows immediately from the above results.

Proposition 2. Suppose that $\mu_{p}<\infty$ for an integer $p \geq 2$.

(1) If $\phi_{Z} \in \Sigma_{1}\left(d_{0}, d_{1}\right)$, then under condition (M.1) we have for $|s| \in\left[m_{n}, M_{n}\right]$

$$
\mathbb{E}|\widehat{N}(s)-N(s)|^{p} \lesssim \frac{1}{|s|^{p}} n^{-\frac{p}{2}+p \kappa_{2}} .
$$

(2) If $\phi_{Z} \in \Sigma_{2}\left(d_{0}, d_{1}, \beta\right)$, then under condition (M.2) we have for $|s| \in\left[m_{n}, M_{n}\right]$

$$
\mathbb{E}|\widehat{N}(s)-N(s)|^{p} \lesssim \frac{1}{|s|^{p}} n^{-\frac{p}{2}+p \theta} .
$$

(3) If $\phi_{Z} \in \Sigma_{3}\left(d_{0}, d_{1}, \beta_{0}, \beta_{1}, \beta, \gamma_{0}, \gamma_{1}\right)$, then under condition (M.3) we have for $|s| \in$ $\left[m_{n}, M_{n}\right]$

$$
\mathbb{E}|\widehat{N}(s)-N(s)|^{p} \lesssim \frac{1}{|s|^{p}} \cdot \max \left(n^{-\frac{p}{2}+p \kappa_{2}},(\alpha \log n)^{\frac{p\left(\beta_{0}-1\right)}{\beta}} n^{-\frac{p}{2}+p \gamma_{0} \alpha}\right) .
$$

Proof. By (2.10) and (2.11) we have

$$
\begin{aligned}
|\widehat{N}(s)-N(s)|^{p} & =\left|\frac{1}{i s}\left(\frac{1}{n \Delta} \sum_{j=1}^{n}\left(Z_{j}-\mathbb{E} Z_{j}\right)-(\widehat{D}(s)-D(s))\right)\right|^{p} \\
& \lesssim \frac{1}{|s|^{p}} \mathbb{E}\left|\frac{1}{n \Delta} \sum_{j=1}^{n}\left(Z_{j}-\mathbb{E} Z_{j}\right)\right|^{p}+\frac{1}{|s|^{p}} \mathbb{E}|\widehat{D}(s)-D(s)|^{p}
\end{aligned}
$$


If $\mu_{p}<\infty$, then $\mathbb{E}\left|Z_{j}\right|^{p}<\infty$. By the Rosenthal inequality we obtain

$$
\mathbb{E}\left|\frac{1}{n} \sum_{j=1}^{n}\left(Z_{j}-\mathbb{E} Z_{j}\right)\right|^{p} \lesssim\left(\frac{1}{n^{p-1}}+\frac{1}{n^{p / 2}}\right) \lesssim \frac{1}{n^{p / 2}} \quad(\text { for } p \geq 2),
$$

which, together with (3.20) and Proposition 1, gives the desired results.

Now we give the main result of this section. For two square integrable functions $f, g$, define the metric $\|f-g\|=\left(\int(f(x)-g(x))^{2} d x\right)^{\frac{1}{2}}$. The following theorem shows consistency of $\widehat{\psi}$ under the risk measure $\mathbb{E}\|\widehat{\psi}-\psi\|^{2}$.

Theorem 1. Suppose that $\mu_{4}<\infty$.

(1) If $\phi_{Z} \in \Sigma_{1}\left(d_{0}, d_{1}\right)$, then under condition (M.1) with $1-3 \kappa_{2}>0$ we have

$$
\mathbb{E}\|\widehat{\psi}-\psi\|^{2} \lesssim \max \left(n^{-1+2\left(\kappa_{1}+\kappa_{2}\right)}, n^{-1+3 \kappa_{2}}, n^{-\kappa_{2}}\right) .
$$

(2) If $\phi_{Z} \in \Sigma_{2}\left(d_{0}, d_{1}, \beta\right)$, then under condition (M.2) with $1-2\left(\kappa_{1}+\theta\right)>0$ and $1-\kappa_{2}-2 \theta>0$ we have

$$
\mathbb{E}\|\widehat{\psi}-\psi\|^{2} \lesssim \max \left(n^{-1+2\left(\kappa_{1}+\theta\right)}, n^{-1+\kappa_{2}+2 \theta}, n^{-\alpha}, n^{-\kappa_{2}}\right) .
$$

(3) If $\phi_{Z} \in \Sigma_{3}\left(d_{0}, d_{1}, \beta_{0}, \beta_{1}, \beta, \gamma_{0}, \gamma_{1}\right)$, then under condition (M.3) with $1-3 \kappa_{2}>0$ we have

$$
\mathbb{E}\|\widehat{\psi}-\psi\|^{2} \lesssim(\alpha \log n)^{-\frac{1}{\beta}} .
$$

Proof. Note that $\psi(u)$ is a real-valued function. Then we have

$$
\begin{aligned}
& \|\widehat{\psi}-\psi\|^{2} \\
\leq & \int_{0}^{\infty}\left|\frac{1}{2 \pi} \int_{m_{n}}^{M_{n}} e^{-i u s} \frac{\widehat{N}(s)}{\widehat{D}(s)} d s+\frac{1}{2 \pi} \int_{-M_{n}}^{m_{n}} e^{-i u s} \frac{\widehat{N}(s)}{\widehat{D}(s)} d s-\psi(u)\right|^{2} d u \\
= & \frac{1}{2 \pi} \int_{m_{n}<|s|<M_{n}}\left|\frac{\widehat{N}(s)}{\widehat{D}(s)}-\frac{N(s)}{D(s)}\right|^{2} d s+\frac{1}{2 \pi} \int_{|s| \geq M_{n}}\left|\frac{N(s)}{D(s)}\right|^{2} d s+\frac{1}{2 \pi} \int_{|s| \leq m_{n}}\left|\frac{N(s)}{D(s)}\right|^{2} d s \\
:= & I I_{1}+I I_{2}+I I_{3},
\end{aligned}
$$

where the second step follows from Parseval's identity. 
For $I I_{1}$, using the inequality $|a+b|^{2} \leq 2|a|^{2}+2|b|^{2}$ and Cauchy-Schwarz inequality we obtain

$$
\begin{aligned}
\mathbb{E} I I_{1} \leq & \mathbb{E} \frac{1}{\pi} \int_{m_{n}<|s|<M_{n}}\left|\frac{\widehat{N}(s)}{\widehat{D}(s)}-\frac{\widehat{N}(s)}{D(s)}\right|^{2} d s+\mathbb{E} \frac{1}{\pi} \int_{m_{n}<|s|<M_{n}}\left|\frac{\widehat{N}(s)}{D(s)}-\frac{N(s)}{D(s)}\right|^{2} d s \\
\leq & \mathbb{E} \frac{2}{\pi} \int_{m_{n}<|s|<M_{n}}|\widehat{N}(s)-N(s)|^{2} \cdot\left|\frac{1}{\widehat{D}(s)}-\frac{1}{D(s)}\right|^{2} d s \\
& +\mathbb{E} \frac{2}{\pi} \int_{m_{n}<|s|<M_{n}}|N(s)|^{2} \cdot\left|\frac{1}{\widehat{D}(s)}-\frac{1}{D(s)}\right|^{2} d s+\mathbb{E} \frac{1}{\pi} \int_{m_{n}<|s|<M_{n}} \frac{|\widehat{N}(s)-N(s)|^{2}}{|D(s)|^{2}} d s \\
:= & I I_{1,1}+I I_{1,2}+I I_{1,3} .
\end{aligned}
$$

For $I I_{2}$ and $I I_{3}$, by Lemma 1 we have

$$
I I_{2}=O\left(M_{n}^{-1}\right), \quad I I_{3}=O\left(m_{n}\right) .
$$

In order to complete the proof, we consider three situations according to the decay rates of $\phi_{Z}$.

First, suppose that $\phi_{Z} \in \Sigma_{1}\left(d_{0}, d_{1}\right)$ and condition (M.1) holds with $1-3 \kappa_{2}>0$. By Cauchy-Schwarz inequality, Proposition 1 and Proposition 2, we have

$$
\begin{aligned}
I I_{1,1} & \leq \frac{2}{\pi} \int_{m_{n}<|s|<M_{n}} \mathbb{E}^{\frac{1}{2}}|\widehat{N}(s)-N(s)|^{4} \cdot \mathbb{E}^{\frac{1}{2}}\left|\frac{1}{\widehat{D}(s)}-\frac{1}{D(s)}\right|^{4} d s \\
& \lesssim \frac{2}{\pi} \int_{m_{n}<|s|<M_{n}}\left(\frac{1}{|s|^{4}} n^{-2+4 \kappa_{2}}\right)^{\frac{1}{2}}\left(\frac{1}{|D(s)|^{4}} n^{-2+4\left(\kappa_{1}+\kappa_{2}\right)}\right)^{\frac{1}{2}} d s \\
& \lesssim n^{-2+2\left(\kappa_{1}+2 \kappa_{2}\right)} \int_{m_{n}}^{M_{n}} \frac{1}{s^{2}} d s \\
& =n^{-2+2\left(\kappa_{1}+2 \kappa_{2}\right)}\left(m_{n}^{-1}-M_{n}^{-1}\right) \\
& \lesssim n^{-2+2 \kappa_{1}+5 \kappa_{2}},
\end{aligned}
$$

where we have used the fact that $|D(s)|>c-\mu_{1}>0$. For $I I_{1,2}$, by Proposition 1 and Lemma 1 we have

$$
\begin{aligned}
I I_{1,2} & \lesssim n^{-1+2\left(\kappa_{1}+\kappa_{2}\right)} \int_{m_{n}<|s|<M_{n}}\left|\frac{N(s)}{D(s)}\right|^{2} d s \\
& \lesssim n^{-1+2\left(\kappa_{1}+\kappa_{2}\right)} \int \frac{1}{1 \vee s^{2}} d s \\
& \lesssim n^{-1+2\left(\kappa_{1}+\kappa_{2}\right)} .
\end{aligned}
$$


For $I I_{1,3}$, by Proposition 3 we have

$$
\begin{aligned}
I I_{1,3} & \lesssim n^{-1+2 \kappa_{2}} \int_{m_{n}}^{M_{n}} \frac{1}{s^{2}} d s \\
& =n^{-1+2 \kappa_{2}}\left(m_{n}^{-1}-M_{n}^{-1}\right) \\
& \lesssim n^{-1+3 \kappa_{2}} .
\end{aligned}
$$

Take $M_{n}=n$. By $(3.25)$ we have

$$
I I_{2}=O\left(n^{-1}\right), \quad I I_{3}=O\left(n^{-\kappa_{2}}\right) .
$$

Combining the above results we find that

$$
\begin{aligned}
\mathbb{E}\|\widehat{\psi}-\psi\|^{2} & \lesssim \max \left(n^{-2+2 \kappa_{1}+5 \kappa_{2}}, n^{-1+2\left(\kappa_{1}+\kappa_{2}\right)}, n^{-1+3 \kappa_{2}}, n^{-1}, n^{-\kappa_{2}}\right) \\
& \lesssim \max \left(n^{-1+2\left(\kappa_{1}+\kappa_{2}\right)}, n^{-1+3 \kappa_{2}}, n^{-\kappa_{2}}\right),
\end{aligned}
$$

since compared with $n^{-1+2\left(\kappa_{1}+\kappa_{2}\right)}$ and $n^{-1+3 \kappa_{2}}, n^{-1+2 \kappa_{1}+5 \kappa_{2}}=n^{-1+2\left(\kappa_{1}+\kappa_{2}\right)} \cdot n^{-1+3 \kappa_{2}}$ and $n^{-1}$ are negligible.

Next, suppose that $\phi_{Z} \in \Sigma_{2}\left(d_{0}, d_{1}, \beta\right)$ and condition (M.2) holds with $1-2\left(\kappa_{1}+\theta\right)>0$ and $1-\kappa_{2}-2 \theta>0$. As in the analysis of the first case, we have

$$
\begin{aligned}
I I_{1,1} & \lesssim \int_{m_{n}}^{M_{n}}\left(\frac{1}{|s|^{4}} n^{-2+4 \theta}\right)^{\frac{1}{2}}\left(\frac{1}{|D(s)|^{4}} n^{-2+4\left(\kappa_{1}+\theta\right)}\right)^{\frac{1}{2}} d s \\
& \lesssim n^{-2+4 \theta+2 \kappa_{1}+\kappa_{2}} \\
I I_{1,2} & \lesssim n^{-1+2\left(\kappa_{1}+\theta\right)} \int_{m_{n}}^{M_{n}}\left|\frac{N(s)}{D(s)}\right|^{2} d s \lesssim n^{-1+2\left(\kappa_{1}+\theta\right)}, \\
I I_{1,3} & \lesssim n^{-1+2 \theta} \int_{m_{n}}^{M_{n}} \frac{1}{s^{2}} d s \lesssim n^{-1+\kappa_{2}+2 \theta}
\end{aligned}
$$

and

$$
I I_{2}=O\left(n^{-\alpha}\right), \quad I I_{3}=O\left(n^{-\kappa_{2}}\right)
$$

Hence,

$$
\begin{aligned}
\mathbb{E}\|\widehat{\psi}-\psi\|^{2} & \lesssim \max \left(n^{-2+4 \theta+2 \kappa_{1}+\kappa_{2}}, n^{-1+2\left(\kappa_{1}+\theta\right)}, n^{-1+\kappa_{2}+2 \theta}, n^{-\alpha}, n^{-\kappa_{2}}\right) \\
& \lesssim \max \left(n^{-1+2\left(\kappa_{1}+\theta\right)}, n^{-1+\kappa_{2}+2 \theta}, n^{-\alpha}, n^{-\kappa_{2}}\right)
\end{aligned}
$$

since compared with $n^{-1+2\left(\kappa_{1}+\theta\right)}$ and $n^{-1+\kappa_{2}+2 \theta}, n^{-2+4 \theta+2 \kappa_{1}+\kappa_{2}}=n^{-1+2\left(\kappa_{1}+\theta\right)} \cdot n^{-1+\kappa_{2}+2 \theta}$ is negligible. 
Finally, suppose that $\phi_{Z} \in \Sigma_{3}\left(d_{0}, d_{1}, \beta_{0}, \beta_{1}, \beta, \gamma_{0}, \gamma_{1}\right)$ and condition (M.3) holds with $1-3 \kappa_{2}>0$. As in the first case, we have

$$
\begin{aligned}
I I_{1,1} \lesssim & \int_{m_{n}}^{M_{n}}\left(\frac{1}{|s|^{4}} \max \left(n^{-2+4 \kappa_{2}},(\alpha \log n)^{\frac{4\left(\beta_{0}-1\right)}{\beta}} n^{-2+4 \gamma_{0} \alpha}\right)\right)^{\frac{1}{2}} \\
& \times\left(\frac{1}{|D(s)|^{4}} \max \left(n^{-2+4\left(\kappa_{1}+\kappa_{2}\right)},(\alpha \log n)^{\frac{4\left(\beta_{0}-1\right)}{\beta}} n^{-2+4\left(\kappa_{1}+\gamma_{0} \alpha\right)}\right)\right)^{\frac{1}{2}} d s \\
\lesssim & \max \left(n^{-1+2 \kappa_{2}},(\alpha \log n)^{\frac{2\left(\beta_{0}-1\right)}{\beta}} n^{-1+2 \gamma_{0} \alpha}\right) \\
& \quad \times \max \left(n^{-1+2\left(\kappa_{1}+\kappa_{2}\right)},(\alpha \log n)^{\frac{2\left(\beta_{0}-1\right)}{\beta}} n^{-1+2\left(\kappa_{1}+\gamma_{0} \alpha\right)}\right) \int_{m_{n}}^{M_{n}} \frac{1}{s^{2}} d s \\
\lesssim & \max \left(n^{-1+3 \kappa_{2}},(\alpha \log n)^{\frac{2\left(\beta_{0}-1\right)}{\beta}} n^{-1+\kappa_{2}+2 \gamma_{0} \alpha}\right) \\
& \quad \times \max \left(n^{-1+2\left(\kappa_{1}+\kappa_{2}\right)},(\alpha \log n)^{\frac{2\left(\beta_{0}-1\right)}{\beta}} n^{-1+2\left(\kappa_{1}+\gamma_{0} \alpha\right)}\right) \\
I I_{1,2} \lesssim & \max \left(n^{-1+2\left(\kappa_{1}+\kappa_{2}\right)},(\alpha \log n)^{\frac{2\left(\beta_{0}-1\right)}{\beta}} n^{-1+2\left(\kappa_{1}+\gamma_{0} \alpha\right)}\right) \int_{m_{n}}^{M_{n}}\left|\frac{N(s)}{D(s)}\right|^{2} d s \\
\lesssim & \max \left(n^{-1+2\left(\kappa_{1}+\kappa_{2}\right)},(\alpha \log n)^{\frac{2\left(\beta_{0}-1\right)}{\beta}} n^{-1+2\left(\kappa_{1}+\gamma_{0} \alpha\right)}\right), \\
I I_{1,3} \lesssim & \max \left(n^{-1+2 \kappa_{2}},(\alpha \log n)^{\frac{2\left(\beta_{0}-1\right)}{\beta}} n^{-1+2 \gamma_{0} \alpha}\right) \int_{m_{n}}^{M_{n}} \frac{1}{s^{2}} d s \\
\lesssim & \max \left(n^{-1+3 \kappa_{2}},(\alpha \log n)^{\frac{2\left(\beta_{0}-1\right)}{\beta}} n^{-1+\kappa_{2}+2 \gamma_{0} \alpha}\right) \\
&
\end{aligned}
$$

and

$$
I I_{2}=O\left((\alpha \log n)^{-\frac{1}{\beta}}\right), \quad I I_{3}=O\left(n^{-\kappa_{2}}\right) .
$$

It is easily seen that under condition (M.3) with $1-3 \kappa_{2}>0$, the rate $(\alpha \log n)^{-\frac{1}{\beta}}$ denominates the other rates. Hence,

$$
\mathbb{E}\|\widehat{\psi}-\psi\|^{2} \lesssim(\alpha \log n)^{-\frac{1}{\beta}}
$$

Remark 3. It follows from Theorem 1 that the convergence rate depends heavily on the decay rate of the characteristic function $\phi_{Z}$. In particular, we obtain convergence rate of polynomial order when $\phi_{Z} \in \Sigma_{1}\left(d_{0}, d_{1}\right)$ or $\phi_{Z} \in \Sigma_{2}\left(d_{0}, d_{1}, \beta\right)$. However, if $\left|\phi_{Z}(s)\right|$ decays at an exponential rate as $|s| \rightarrow \infty$, only logarithmic convergence rate can be obtained. 
When estimating the Lévy density, the polynomial decay rate of $\phi_{Z}$ leads to polynomial convergence rate of the estimator, and the exponential decay rate of $\phi_{Z}$ usually leads to logarithmic convergence rate. See Neumann and Reiß(2009), Kappus and Reiß(2010) and Kappus (2014). Hence, we obtain the same conclusion when estimating the ruin probability in the Lévy risk model.

\section{Simulation studies}

In this section we provide some simulation results to illustrate the behavior of our estimator when the sample size is finite. We use fast Fourier (inversion) transform to compute the estimator.

By (2.12), we have $\widehat{\psi}(u)=\mathcal{R} \zeta(u)$, where

$$
\begin{aligned}
\zeta(u) & =\frac{1}{2 \pi} \int_{m_{n}}^{M_{n}} e^{-i u s} \frac{\widehat{N}(s)}{\widehat{D}(s)} d s+\frac{1}{2 \pi} \int_{-M_{n}}^{-m_{n}} e^{-i u s} \frac{\widehat{N}(s)}{\widehat{D}(s)} d s \\
& :=\zeta_{1}(u)+\zeta_{2}(u) .
\end{aligned}
$$

For a small constant $a>0$, we can approximate $\zeta_{1}(u)$ as follows,

$$
\zeta_{1}(u) \approx \sum_{j=1}^{K} e^{-i u a(j-1)} \frac{a \widehat{N}((j-1) a)}{2 \pi \widehat{D}((j-1) a)} \mathbf{1}_{\left(m_{n} \leq(j-1) a \leq M_{n}\right)},
$$

where $K$ is taken to be some power of 2 . In particular, for

$$
u_{k}=\frac{2 \pi}{a K}(k-1), \quad k=1,2, \ldots, K,
$$

we have

$$
\zeta_{1}\left(u_{k}\right) \approx \sum_{j=1}^{K} \exp \left(-\frac{2 \pi i}{K}(j-1)(k-1)\right) \frac{a \widehat{N}((j-1) a)}{2 \pi \widehat{D}((j-1) a)} \mathbf{1}_{\left(m_{n} \leq(j-1) a \leq M_{n}\right)} .
$$

Then we can use fast Fourier transform to compute $\zeta_{1}$. As for $\zeta_{2}$, we have

$$
\zeta_{2}(u) \approx \frac{1}{K} \sum_{j=1}^{K} e^{i u a(j-1)} \frac{a K \widehat{N}((1-j) a)}{2 \pi \widehat{D}((1-j) a)} \mathbf{1}_{\left(m_{n} \leq(j-1) a \leq M_{n}\right)} .
$$

In particular, for $u_{k}, k=1,2, \ldots, K$, we have

$$
\zeta_{2}\left(u_{k}\right) \approx \frac{1}{K} \sum_{j=1}^{K} \exp \left(\frac{2 \pi i}{K}(j-1)(k-1)\right) \frac{a K \widehat{N}((1-j) a)}{2 \pi \widehat{D}((1-j) a)} \mathbf{1}_{\left(m_{n} \leq(j-1) a \leq M_{n}\right)},
$$


which implies that we can apply fast Fourier inversion transform to compute $\zeta_{2}$.

We assume that the characteristic exponent is given by

$$
\Psi(s)=i c s-\frac{\sigma^{2}}{2} s^{2}+\int_{0}^{\infty}\left(e^{-i s x}-1\right) \lambda \mu e^{-\mu x} d x,
$$

where $c, \lambda, \mu>0$ and $c>\lambda / \mu$. When $\sigma=0, X$ is a classical risk model with exponential jumps, and the ruin probability is given by

$$
\psi(u)=\frac{\lambda}{c \mu} e^{-\left(\mu-\frac{\lambda}{c}\right) u}, \quad u \geq 0 .
$$

When $\sigma>0, X$ is a compound Poisson model perturbed by diffusion, and the ruin probability is given by

$$
\psi(u)=\frac{R_{1}+\mu+\frac{2 \lambda}{\mu \sigma^{2}}}{R_{1}-R_{2}} e^{R_{1} u}+\frac{R_{2}+\mu+\frac{2 \lambda}{\mu \sigma^{2}}}{R_{2}-R_{1}} e^{R_{2} u}, \quad u \geq 0,
$$

where $R_{2}<R_{1}<0$ are negative roots of the following equation (in $s$ )

$$
\frac{1}{2} \sigma^{2} s+c-\frac{\lambda}{s+\mu}=0 .
$$

In the following simulation, we set $c=1.2, \lambda=1, \mu=1, \Delta=1, a=0.001$ and $K=2^{16}$. When $X$ is a compound Poisson model, we have $\phi_{Z} \in \Sigma_{1}\left(d_{0}, d_{1}\right)$ with $d_{1}=1$ and $d_{0}=e^{-2 \lambda}=e^{-2}$. See Example 1 in Section 3. We choose $r_{n}=0.01 \times n^{-1 / 3}$, $m_{n}=0.01 \times n^{-1 / 3}$ by hand. When $X$ is a compound Poisson model perturbed by diffusion, we set $\sigma^{2}=1$. It follows from Example 4 in Section 3 that $\phi_{Z} \in \Sigma_{3}\left(d_{0}, d_{1}, \beta_{0}, \beta_{1}, \beta, \gamma_{0}, \gamma_{1}\right)$ with $d_{0}=e^{-2}, d_{1}=1, \beta_{0}=\beta_{1}=0, \gamma_{0}=\gamma_{1}=\frac{1}{2}, \beta=2$. In this case, we choose $r_{n}=0.01 \times n^{-1 / 5}, m_{n}=0.01 \times n^{-1 / 5}$ and $M_{n}=100 \times(0.2 \log n)^{1 / 2}$ by hand.

In Figure 1 (a), we plot the true ruin probability curve and 20 estimated curves with sample size $n=1000$. We find that the estimates have larger volatility when the curvature is large ( for $u \in[5,20]$ ). This implies that ruin probability is hard to estimate when the curve is complex. In Figure 1 (b), we plot the true ruin probability curve and some mean curves with sample sizes $n=200,500,1000,3000$, which are computed based on 500 simulation experiments. As is expected, the results improve as the sample size improves. Likewise, we illustrate some simulation results in Figure 2 when $X$ is a compound Poisson model perturbed by diffusion. We can obtain the same conclusions as in the compound Poisson model. Furthermore, comparing Figure 1 with Figure 2, we find that the ruin probability is harder to estimate when the diffusion volatility exists. This is in agreement with the results in Theorem 1. Finally, we compute the mean squared errors and present some results in Table 1 and Table 2. The results are computed based on the above 500 experiments. We find that the mean squared errors decrease w.r.t. the sample size. Again, comparing the values of the same cells across these tables, we observe that the ruin probability is harder to estimate when diffusion volatility exists. 


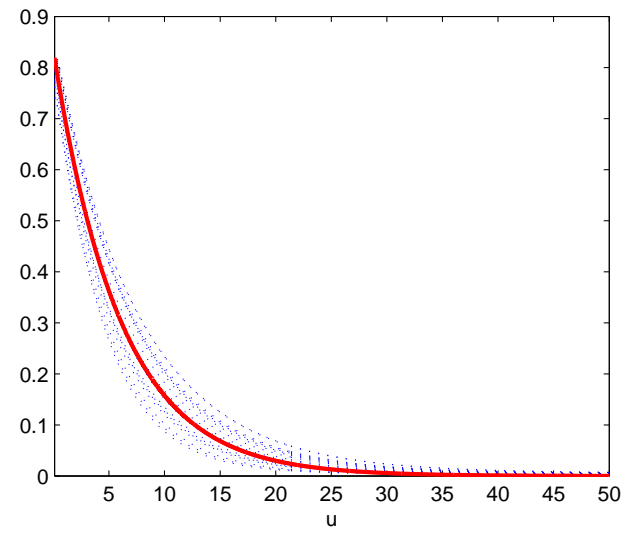

(a)

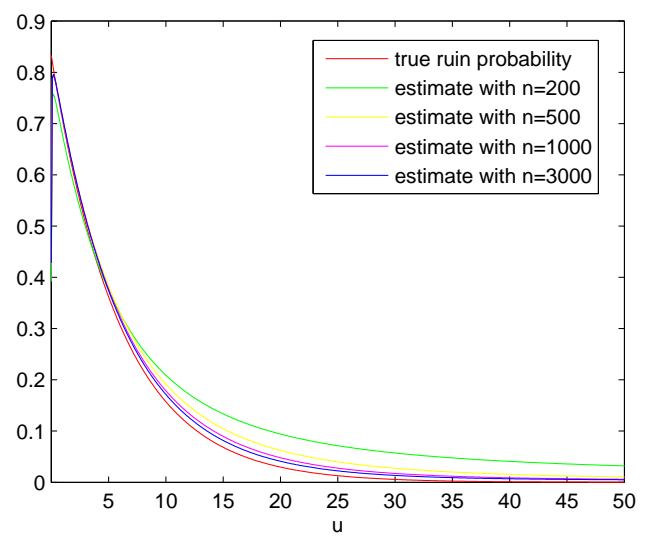

(b)

Figure 1: Estimation of the ruin probability in the compound Poisson model. (a) True curve (bold red line) and 20 estimated curves (dotted blue lines) with sample size $n=1000$; (b) True curve and mean curves with sample sizes $n=200,500,1000,3000$.

Table 1: Mean squared errors for the estimates when $X$ is a compound Poisson model.

\begin{tabular}{lllllllllll}
\hline & $u_{10}$ & $u_{20}$ & $u_{30}$ & $u_{40}$ & $u_{50}$ & $u_{60}$ & $u_{70}$ & $u_{80}$ & $u_{90}$ & $u_{100}$ \\
\hline$n=200$ & 0.0333 & 0.0379 & 0.0419 & 0.0448 & 0.0465 & 0.0472 & 0.0473 & 0.0468 & 0.0460 & 0.0450 \\
$n=500$ & 0.0073 & 0.0118 & 0.0154 & 0.0179 & 0.0193 & 0.0199 & 0.0199 & 0.0195 & 0.0187 & 0.0178 \\
$n=1000$ & 0.0034 & 0.0056 & 0.0073 & 0.0084 & 0.0089 & 0.0091 & 0.0089 & 0.0084 & 0.0079 & 0.0072 \\
$n=3000$ & 0.0012 & 0.0020 & 0.0027 & 0.0031 & 0.0033 & 0.0033 & 0.0032 & 0.0031 & 0.0028 & 0.0026 \\
\hline
\end{tabular}

\section{Appendix A. Useful inequalities}

We present two useful inequalities that are frequently used in this paper.

Bernstein's inequality. Let $Y_{1}, \ldots, Y_{n}$ be independent random variables with zero means and bounded ranges: $\left|Y_{j}\right| \leq M(j=1, \ldots, n)$. If $\sum_{j=1}^{n} \operatorname{Var}\left(Y_{j}\right) \leq V$, then for each $\eta>0$

$$
\mathbb{P}\left(\left|\sum_{j=1}^{n} Y_{j}\right| \geq \eta\right) \leq 2 \exp \left(-\frac{\frac{1}{2} \eta^{2}}{V+\frac{1}{3} M \eta}\right)
$$

Table 2: Mean squared errors for the estimates when $X$ is a perturbed compound Poisson model.

\begin{tabular}{lllllllllll}
\hline & $u_{10}$ & $u_{20}$ & $u_{30}$ & $u_{40}$ & $u_{50}$ & $u_{60}$ & $u_{70}$ & $u_{80}$ & $u_{90}$ & $u_{100}$ \\
\hline$n=200$ & 0.0508 & 0.0498 & 0.0507 & 0.0522 & 0.0532 & 0.0540 & 0.0546 & 0.0545 & 0.0543 & 0.0539 \\
$n=500$ & 0.0137 & 0.0162 & 0.0197 & 0.0226 & 0.0248 & 0.0270 & 0.0285 & 0.0294 & 0.0302 & 0.0303 \\
$n=1000$ & 0.0027 & 0.0047 & 0.0068 & 0.0088 & 0.0104 & 0.0116 & 0.0124 & 0.0129 & 0.0131 & 0.0131 \\
$n=3000$ & 0.0012 & 0.0020 & 0.0028 & 0.0036 & 0.0042 & 0.0046 & 0.0049 & 0.0050 & 0.0050 & 0.0050 \\
\hline
\end{tabular}




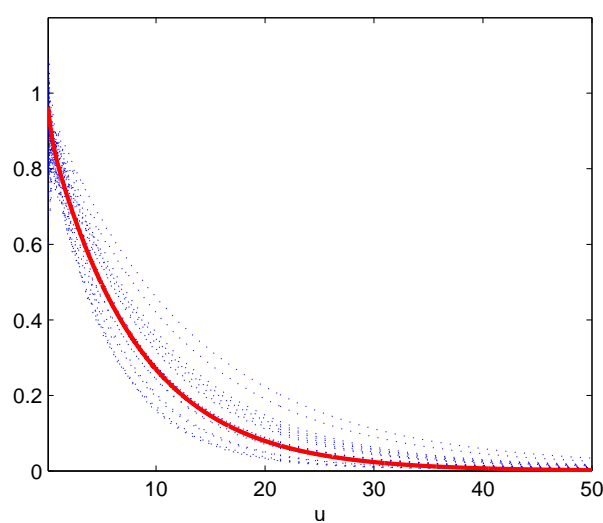

(a)

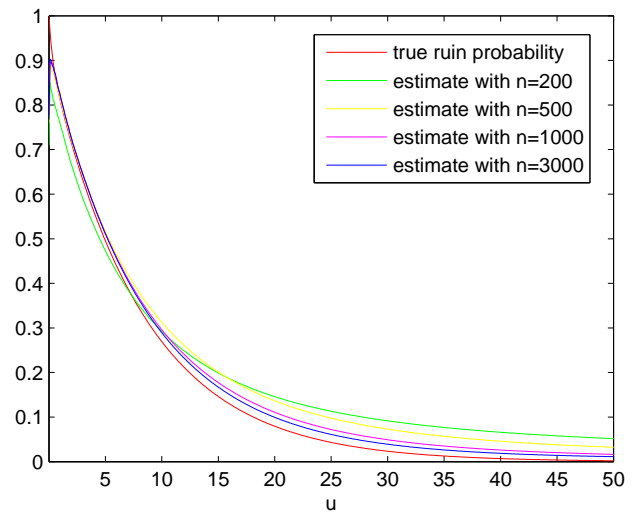

(b)

Figure 2: Estimation of the ruin probability in the compound Poisson model perturbed by diffusion. (a) True curve (bold red line) and 20 estimated curves (dotted blue lines) (b) True curve and mean curves with sample sizes $n=200,500,1000,3000$.

The Rosenthal inequality. Let $Y_{1}, \ldots, Y_{n}$ be independent random variables with zero means, such that $\mathbb{E}\left|Y_{j}\right|^{p}<\infty(j=1, \ldots, n)$ for an integer $p \geq 1$. Then there exists a constant $C$ depending only on $p$ such that

$$
\mathbb{E}\left|\sum_{j=1}^{n} Y_{j}\right|^{p} \leq C \cdot\left(\sum_{j=1}^{n} \mathbb{E}\left|Y_{j}\right|^{p}+\left(\sum_{j=1}^{n} \mathbb{E} Y_{j}^{2}\right)^{p / 2}\right) .
$$

\section{Acknowledgements}

The authors would like to thank the anonymous referees for their helpful comments and suggestions, which improved an earlier version of the paper. Zhimin Zhang is supported by the National Natural Science Foundation of China (11101451) and the Natural Science Foundation Project of CQ CSTC of China (cstc2014jcyjA00007). Hailiang Yang acknowledges the support from the Research Grants Council of the Hong Kong Special Administrative Region (project No. HKU 705313P) and Society of Actuaries' Centers of Actuarial Excellence Research Grant.

\section{References}

[1] Asmussen, S., Albrecher, H., 2010. Ruin Probabilities, Second Edition. World Scientific, Singapore.

[2] Croux, K., Vervaerbeke, N., 1990. Nonparametric estimators for the probability of ruin. Insurance: Mathematics and Economics 9(2-3): 127-130. 
[3] Chung, K.L., 2001. A Course in Probability, Third Edition. Academic Press, San Diego.

[4] Comte, F., Genon-Catalot, V., 2009. Nonparametric estimation for pure jump Lvy processes based on high frequency data. Stochastic Processes and their Applications 119(12), 4088-4123.

[5] Comte, F., Genon-Catalot, V., 2010. Nonparametric adaptive estimation for pure jump Lévy processes. Annales de l'Institut Henri Poincaré, Probabilités et Statistiques 46(3), 595-617.

[6] Comte, F., Genon-Catalot, V., 2010. Estimation for Lévy processes from high frequency data within a long time interval . Annals of Statistics 39(2), 803-837.

[7] Fan, J., 1991. On the optimal rates of convergence for nonparametric deconvolution problems. The Annals of Statistics 19, 1257-1272.

[8] Frees, E.W., 1986. Nonparametric estimation of the probability of ruin. ASTIN Bulletin 16, 81-90.

[9] Gugushvili, S., 2009. Nonparametric estimation of the characteristic triplet of a discretely observed Lévy process. Journal of Nonparametric Statistics 21(3), 321-343.

[10] Gugushvili, S., 2012. Nonparametric estimation for discretely sampled Lévy processes. Annales de l'Institut Henri Poincaré, Probabilités et Statistiques 48(1), 282 307.

[11] Hipp, C., 1989. Estimators and bootstrap confidence intervals for ruin probabilities. ASTIN Bulletin 19, 57-70.

[12] Huzak, M., Perman, M., Šikić, H., Vondraček, Z., 2004. Ruin probabilities and decompositions for general perturbed risk processes. The Annals of Applied Probability 14(3), 1378-1397.

[13] Kappus, J., 2014. Adaptive nonparametric estimation for Lvy processes observed at low frequency. Stochastic Processes and Their Applications 124(1), 730-758.

[14] Kappus, J., Reiß, M., 2010. Estimation of the characteristics of a Lvy process observed at arbitrary frequency. Statistica Neerlandica 64(3), 314-328.

[15] Masiello, E., 2012. On semiparametric estimation of ruin probabilities in the classical risk model. Scandinavian Actuarial Journal 2014(4), 283-308.

[16] Neumann, M.H., and Markus Reiß, M., 2012. Nonparametric estimation for Lévy processes from low-frequency observations. Bernoulli 15(1), 223-248. 
[17] Pitts, S.M., 1994. Non-parametric estimation of compound distributions with applications in insurance. Annals of the Institute of Statistical Mathematics 46(3), 537-555.

[18] Politis, K., 2003. Semiparametric estimation for non-ruin Probabilities. Scandinavian Actuarial Journal 2003(1), 75-96.

[19] Shimize, Y., 2009. A new aspect of a risk process and its statistical inference. Insurance: Mathematics and Economics 44(1), 70-77.

[20] Shimize, Y., 2012. Non-parametric estimation of the Gerber-Shiu function for the Wiener-Poisson risk model. Scandinavian Actuarial Journal 2012(1), 56-69.

[21] Zhang, Z., Yang, H., Yang, H., 2012. On a nonparametric estimator for ruin probability in the classical risk model. Scandinavian Actuarial Journal 2014(4), 309-338.

[22] Zhang, Z., Yang, H., 2013. Nonparametric estimate of the ruin probability in a purejump Lévy risk model. Insurance: Mathematics and Economics 2013(53), 24-35. 